\title{
A Review of Studies Assessing Cannabidiol's (CBD) Therapeutic Action and Potentials in Respiratory Diseases
}

\author{
Arwa Fairaq ${ }^{1}$, Sahar El-Ashmony ${ }^{1,2}$, Yosra AL-Hindi ${ }^{1}$ \\ ${ }^{I}$ Assistant Professor in Clinical Pharmacy, Faculty of Pharmacy, University of Umm Al-Qura, Makkah, KSA, \\ ${ }^{2}$ Faculty of Medicine, Medical Pharmacology Department, Cairo University, Cairo, Egypt
}

\begin{abstract}
Cannabidiol (CBD) is the major non-mind-altering section of the hairy glands of hemp plant that can be named 'cannabinoids' (CBs). Cannabinoid in the pharmacology field is becoming an extensive subject specifically towards the therapeutic characteristics of cannabinoid receptor agonists. Examples such as analgesia, muscle relaxation, immunosuppressive, antiinflammatory and anti-allergic effects, mood improvement, appetite stimulation, antiemesis, lowering of intraocular pressure, bronchodilatation, neuroprotection, and anti-neoplastic effects. Although this vast knowledge about the therapeutic properties of CBs, their clinical uses remains a controversy and very limited. However, the focus now has been on CBD that constitutes up to $40 \%$ of the extract of the Cannabis and represents one of the most attractive prospects for therapeutic use owing to its extraordinary lack of both perceptual and psychoactive behavior. Finally, CBD is regarded as an important putative lead compound to produce cannabinomimetic drugs because of its excellent tolerability in humans.
\end{abstract}

Keywords: CBD, Respiratory, pharmacology, review.

\section{Introduction}

Cannabidiol: The terms cannabis and marijuana are frequently used interchangeably, but Cannabis is a generic term that includes cannabinoids, marijuana, and hemp-derived from the plant Cannabis sativa. The documented use of cannabis dates to several centuries $\mathrm{BC}^{1}$. The cannabis plant and its derivatives have been exploited for centuries for recreational and medicinal purposes, with millions of regular users around the world. Legislation around these purposes is increasingly considered by local, regional, and national governments ${ }^{1}$. Non-psychotropic Phyto-cannabinoid CBD is considered

\section{Corresponding Author:}

\section{Dr. Yosra Alhindi}

Assistant Professor in Clinical Pharmacy Department, Umm Alqura University, Makkah, Saudi Arabia

Mobile No.: 00966504530609

e-mail: yzhindi@uqu.edu.sa one of the most interesting emerging molecules in the field of pharmacology since it exerts a wide range of therapeutic effects, ranging from anticonvulsive, sedative, hypnotic, antipsychotic, anti-cancer, antiinflammatory and neuroprotective activities ${ }^{2}$.

More than 100 different cannabinoids have been identified, but delta-9-tetrahydrocannabinol (THC) is the most responsible fort he psychoactive effects of euphoria and relaxation. Cannabinoid 1 (CB1) receptors in the brain correlate with the psychoactive effects ${ }^{3}$. CBD is suggested to have therapeutic potential in many disorders, including inflammation, oxidative stress, cancer, diabetes, gastrointestinal disturbances, neurodegenerative disorders, and nociception ${ }^{4,5}$. Evidence is also now accumulating that there are beneficial effects of CBD in the vasculature. A review of the safety and side effects of CBD concluded that CBD appears to be well tolerated at high doses and with chronic use in humans, and thus has the potential to betaken safely into the clinic. Indeed, CBD is one of the active ingredients of the currently licensed medication, Sativex ${ }^{6}$. 


\section{CBD in some respiratory diseases:}

Asthma and COPD: Asthma is a chronic inflammatory disorder of the airways with an increase in the bronchial hyperresponsiveness to different stimuli. Characterized by wheezing, breathlessness, chest tightness, cough, and reversible airflow obstruction ${ }^{7}$. Chronic obstructive pulmonary disease (COPD) is another respiratory disease that can be overlapped with asthma. COPD characterized by chronic airflow limitation, usually caused by significant exposure to noxious particles ${ }^{7}$. COPD, including Chronic Bronchitis and Emphysema, are Cause of death rank 4 with an expectation to increase in coming decades due to continued exposure to different COPD risk factors such as smoking, aging, and inactivity ${ }^{8}$. Treatments include bronchodilators, antimuscarinic agents, corticosteroids, and antiinflammatory medications are available. However, there is no cure for Asthma or COPD until now 9 .

In the last decays, Cannabis, with its different content including Cannabidiol (CBD), draws attention as some studies show its variable pharmacological properties, including anti-inflammatory ${ }^{10}$ immunomodulatory, and analgesic properties. Mechanistically via activation of cannabinoid-1 and -2 (CB1 and $\mathrm{CB} 2$ ) receptors presented predominantly in the central nervous system (CNS) and immune cells, respectively ${ }^{10}$. In immune cells, cannabinoids dysregulate cytokine leading to disruption of the immune system through ${ }^{11}$. Most studies explored the properties of Cannabis, but we're not focusing specifically on CBD. Therefore, further studies were conducted to explore the specific effect of CBD in lung disease. Studying genes related to respiratory disease COPD, Cannabis affected the expression of proinflammatory genes in human small airway epithelial cells (HSAEpC), in vitro ${ }^{12}$.

In vivo, CBD $(20 \mathrm{mg} / \mathrm{kg})$ administration in lipopolysaccharide (LPS) induced lung injury murine and mice model decreased the migration of leukocyte into the lungs, albumin concentration in the bronchoalveolar lavage fluid, as well as production of pro-inflammatory cytokines (TNF and IL-6) and chemokines (MCP-1 and MIP-2) via adenosine $A_{2 A}$ receptor ${ }^{13}$. In an asthmatic rat model, $\mathrm{CBD}$ at the dose of $5 \mathrm{mg} / \mathrm{kg}$ intraperitoneally (i.p) once daily significantly decreased inflammatory response by decreasing the level of both Th1 including TNF- $\alpha$ and IL- 6 as well as Th2 cytokines including IL3, IL-4, IL-5, IL-13 and IL-10 ${ }^{13}$. Furthermore, CBD treatment improved remodeling processes and reduced airway inflammation and fibrosis. Specifically, CBD decreased the collagen fiber content (CBD 5 or $10 \mathrm{mg} /$ $\mathrm{kg}$ i.p.) and lung elastance (CBD $10 \mathrm{mg} / \mathrm{kg}$ i.p.) through $\mathrm{CB} 1 / \mathrm{CB} 2$ signaling ${ }^{14}$. In addition, $\mathrm{CBD}$ improves pulmonary function as its administration associated with an increase in tidal volume, inspiratory flow rate, and respiration rate in the African Green Monkey model with induced pulmonary fibrosis ${ }^{15}$.

The potential therapeutic effects of CBD in lung cancer: The searching to ameliorate the prognosis of lung cancer has led to the development and evaluation of new drugs with mechanisms of action that vary from those of conventional chemotherapeutics used for many years worldwide. A major attempt is now being placed in developing and assessing the potential of targeted therapies and immunotherapy in lung cancer that cause improvement in the clinical outcomes ${ }^{16}$. Thus, targeted therapy is replacing conventional chemotherapy as a standard treatment for patients with targetable oncogenic drivers ${ }^{16}$. However, it has to be recognized that responses to these agents are still partial, with tumors recurring during follow-up. In fact, due to tumors' genetic heterogeneity, a complete response in lung cancer patients is very difficult to attain ${ }^{17}$. The challenge to proceed with the outcome of patients with lung cancer is leading to the assessment of alternative drugs, which, alone or in combination, may lead to improved response and survival in patients. CBD is one possible example as it could be a potential drug in the treatment of cancer. CBD has been shown to have anti-neoplastic effects in vitro and/or in vivo in lung cancerand other types of cancer ${ }^{17,18}$. Newly, the therapeutic potential of the cannabis plant in the area of cancer treatment has been discovered, and the use of Cannabis by cancer patients has increased significantly ${ }^{19}$. The beneficial effects of Cannabis are related to symptoms of the disease: pain, nausea and vomiting, loss of appetite, weight loss, mood swings, and sleep disorders ${ }^{19}$. Also, Cannabis was found to have immunomodulatory properties. Researches assaying the effects of cannabinoid-based drugs on immunity have shown that various cellular and cytokine mechanisms are suppressed by these agents, mainly by four mechanisms: induction of apoptosis (of $\mathrm{T}$ cells, macrophages, splenocytes, and thymocytes), inhibition of cell proliferation, inhibition of production of chemokines and cytokines, and induction of T-regs ${ }^{19}$. Possible clinical use of cannabinoids for the treatment of extremely invasive cancer types is highly supported 
by studies proving a decrease of tumor cell invasion by them ${ }^{19}$. Moreover, the lack of severe adverse effects of cannabinoids as compared to the generalized toxicity of conventional chemotherapies. Furthermore, several major side effects of chemotherapeutics, such as emesis and inherent toxicity on noncancerous tissues, have been demonstrated to be even reduced on treatment with cannabinoids ${ }^{17}$. Within cannabinoid-based substances, the Phytocannabinoid CBD has protruded as a particularly interesting drug due to its lack of adverse psychoactive effects, as well as its considerable antitumorigenic properties ${ }^{17,18,19}$.

It is postulated that CBD could act on tumor cells, directly or indirectly, through different pathways that may vary in different tumor cells. CBD is an inverse agonist for the $\mathrm{CB} 2$ receptor and an antagonist for the $\mathrm{CB} 1$ receptor $^{18,}{ }^{19}$. In addition, $\mathrm{CBD}$ has anti-cancer effects acting as an agonist for the transient receptor potential vanilloid (TRPV) 1 and 2, leading to changes in intracellular $\mathrm{Ca} 2+$ levels $^{19}$. It is also reported that $\mathrm{CBD}$ can induce apoptosis in cancer cells via the production of reactive oxygen species (ROS), caspase activation and activation of p53 dependent apoptotic pathways in cancer cells and down-regulation of mammalian target of rapamycin (mTOR) and cyclin $\mathrm{D} 1^{19}$. CBD can also upregulate TNF/TNFR1 and TRAIL/ TRAIL-R2 signaling by modulation of both ligand and receptor levels, followed by apoptosis. Furthermore, CBD inhibits human umbilical vein endothelial cells (HUVEC) migration, invasion and sprouting in vitro, and angiogenesis in vivo through down-modulation of several angiogenesis-related molecules. From the immunological point of view, CBD significantly inhibits the recruitment of tumor-associated macrophages (TAM) in primary tumor stroma and secondary lung metastases. CBD promotes the susceptibility of cancer cells to adhere to and subsequently be lysed by Lymphokine Activated Killer (LAK) cells, with both effects being reversed by a neutralizing ICAM-1 antibody ${ }^{19}$.

A study used different human lung cancer cell lines, primary tumor cells, and an in vivo intravenous metastasis model, to analyze the contribution of ICAM1 to the anti-invasive action of CBD. This has been previously described to be mediated via cannabinoid receptor-, TRPV1-, and MAPK-dependent upregulation of TIMP-1 in human cervical and lung cancer cells. Accumulating evidence highly supported the role of ICAM-1 as a functional link between cannabinoid receptor- and TRPV1-elicited p42/44 MAPK activation and downstream TIMP-1-dependent inhibition of invasion. First, antagonists to $\mathrm{CB} 1$ and $\mathrm{CB} 2$, as well as an antagonist to TRPV1, were shown to inhibit CBDinducedICAM-1 expression, whichisinlinewithCB D's established cannabinoid- and TRPV1-dependent up-regulation of TIMP-1 expression and subsequent anti-invasive action. Second, CBD induced ICAM-1 expression was found to occur via a receptor-dependent activation of p42/44 MAPK - a pathway likewise shared by the published TIMP-1-dependent anti-invasive action by this cannabinoid. Third, in all cell lines, as well as primary tumor cells from a brain metastasis of a patient with non-small cell lung cancer (NSCLC), CBD elicited a sequential up-regulation of both anti-invasive mediators, ICAM- 1 and TIMP- $1{ }^{19}$.

\section{Cannabidiol as a potential antiinflammatory treatment in SARS-CoV-2 infection}

SARS-CoV-2 is a novel beta coronavirus first reported in China with a 14-day incubation period ${ }^{20}$. In a proportion of individuals, critical illness develops and is characterized by respiratory failure, shock, and multi-organ dysfunction ${ }^{20}$. Mortality ratingalters based on the study population examined, with the death rateamongpersons requiring mechanical ventilation as high as $88 \%^{20}$. SARS-CoV-2 infects types I and II pn eumocytesviaitsreceptorangiotensin-convertingenzyme (ACE)2, which is also them a in receptor for SARS$\mathrm{CoV}-2^{20}$. Under healthy circumstances, bronchoalveolar lavagefluidismadeup of predominantly alveolar macro phages $(<80 \%)$ and lymphocytes $(\sim 10-20 \%)$. Alveolar macrophages control the lungs for pathogens, clears senescent cells, shareinreparation of -damagedtissue, andenhance $\mathrm{T}$-cellspecificresponses ${ }^{20}$. Importantly, SARS-CoV-2 infection causes hyper-activation of lung macrophages as well as marked infiltration of proinflammatory monocyte-derived macrophages (MDMs) into small airways. Acute macrophage stimulation initiates a massive pro-inflammatory response, including IL-6 and IL-1 $\beta$, which enhance the recruitment of neutrophils and cytotoxic CD8T-cells into the lung's mucosal tissues ${ }^{21}$.

Cannabinoids can suppress immune activation and inflammatory cytokine production, suggesting their potential for alleviating excessive inflammation. Endocannabinoid receptors include CB1andCB2. CB1 has higher expression in the central nervous system and a lesser expression on peripheral tissues, including the lungs ${ }^{22}$. CB2 is expressed by lungs as well as varieties 
of immune cells, including circulating lymphocytes, monocytes, and tissue mast cells and in lymphoid tissues. Activation of the $\mathrm{CB} 2$ receptor can inhibit the release of inflammatory IL-1, IL-6, IL-12, and TNF- $\alpha$. Constitutive production of endocannabinoids occurs by human lung resident macrophages, which is protective in acute and chronic inflammation, mostly via CB2 receptors ${ }^{21}$. Agonists of CB2 have been shown to inhibit TNF- $\alpha$ from CD14+ monocytes and M1 macrophages and increase expression of antiinflammatory cytokine IL10. CB2 agonists also induce antiinflammatory FoxP3+ regulatory T-cells (Tregs), which produce TGF- $\beta$ and IL-10. In addition, CBD has been shown to induce the differentiation of functional immunosuppressive Tregs ${ }^{22}$.

In humans, CBD has been tested across a wide dosage range, varying from $<1$ up to $50 \mathrm{mg}$ pro $\mathrm{kg} / \mathrm{die}$ depending on the trials and on the explored pathological condition, with both in vitro and in vivo studies suggesting an immunosuppressive action at higher concentrations or doses ${ }^{22}$. CBD should be given orally starting at 100 $\mathrm{mg}$ /day titrating up to $300 \mathrm{mg} /$ day $(2.5 \mathrm{mg} / \mathrm{kg} / \mathrm{die})$ since this dosage did not produce relevant adverse effects even for prolonged administrations (up to 18 weeks) in clinical trials in humans. CBD acts as an in vitro inhibitor of several CYP450 isoforms. Since drug-drug interaction studies between CBD and anti-COVID-19 treatments are lacking; therefore, monitoring of patients for potential drug interactions would be required ${ }^{22}$. It is concluded that SARS-CoV2 causes severe damage through the cytokine storm that causes inflammation by macrophages and other immune cells. Since the CBD has broad antiinflammatory properties, it may represent a potential antiinflammatory therapeutic approach against SARS-CoV2, and it is indeed a therapeutic agent used in clinical medicine and has a favorable safety profile.

\section{Conclusion}

Cannabidiol elucidates an impressive plethora of actions, specifically as an anti-inflammatory agent. Many of which could have medicinal significance as well as the lead in the production of pharmaceuticals. CBD is a compound well tolerated in humans with a very low toxicity profile and devoid of psychoactive and cognitive effects. However, more clinical trials are needed to be able to validate its beneficial properties as the possible results that can be discovered by these trials can give a lot of progress from the present preclinical evidence to a practical therapeutic application.

\section{Conflict of Interest: None}

Ethical Clearance: Taken from IRB committee of umm Alqura university, Makkah, KSA.

\section{Source of Support: Nill}

\section{References}

1. Long T, Wagner M, Demske D, Leipe C, Tarasov PE. Cannabis in Eurasia: origin of human use and Bronze Age trans-continental connections. Vegetation History and Archaeobotany. 2017 Mar;26(2):245-58..

2. Machado Bergamaschi M, Helena Costa Queiroz R, Waldo Zuardi A, Crippa AS. Safety and side effects of cannabidiol, a Cannabis sativa constituent. Current drug safety. 2011 Sep 1;6(4):237-49.

3. Jett J, Stone E, Warren G, Cummings KM. Cannabis use, lung cancer, and related issues. Journal of Thoracic Oncology. 2018 Apr 1;13(4):480-7.

4. Ladin DA, Soliman E, Griffin L, Van Dross R. Preclinical and clinical assessment of cannabinoids as anti-cancer agents. Frontiers in pharmacology. 2016 Oct 7;7:361.

5. Pertwee RG. The diverse CB1 and CB2 receptor pharmacology of three plant cannabinoids: $\Delta$ 9-tetrahydrocannabinol, cannabidiol and $\Delta 9$-tetrahydrocannabivarin. British journal of pharmacology. 2008 Jan;153(2):199-215.

6. Esposito G, Scuderi C, Valenza M, Togna GI, Latina V, De Filippis D, Cipriano M, Carratù MR, Iuvone $\mathrm{T}$, Steardo L. Cannabidiol reduces $A \beta$-induced neuroinflammation and promotes hippocampal neurogenesis through PPAR $\gamma$ involvement. PloS one. 2011 Dec 5;6(12):e28668.

7. Tat TS, Cilli A. Evaluation of long-term safety and efficacy of omalizumab in elderly patients with uncontrolled allergic asthma. Annals of Allergy, Asthma \& Immunology. 2016 Nov 1;117(5):546-9.

8. Zeind CS, Carvalho MG, editors. Applied Therapeutics: The Clinical Use of Drugs. Wolters Kluwer; 2018.

9. Singh D, Agusti A, Anzueto A, Barnes PJ, Bourbeau J, Celli BR, Criner GJ, Frith P, Halpin DM, Han M, Varela MV. Global strategy for the diagnosis, management, and prevention of chronic obstructive lung disease: the GOLD science committee report 2019. European Respiratory Journal. 2019 May $1 ; 53(5)$. 
10. Centers for Disease Control and Prevention. Leading Causes of Death. https://www.cdc. gov/nchs/fastats/leading-causes-of-death.htm. (Accessed on July 05, 2020).

11. Mathers CD, Loncar D. Projections of global mortality and burden of disease from 2002 to 2030 . PLoS medicine. 2006 Nov 28;3(11):e442.

12. Król A, Palmér R, Rondeau V, Rennard S, Eriksson $\mathrm{UG}$, Jauhiainen A. Improving the evaluation of COPD exacerbation treatment effects by accounting for early treatment discontinuations: a post-hoc analysis of randomized clinical trials. Respiratory research. 2020 Dec;21(1):1-1.

13. de Freitas Cuba L, Salum FG, Guimarães FS, Cherubini K, Borghetti RL, de Figueiredo MA. Cannabidiol on 5-FU-induced oral mucositis in mice. Oral Diseases. 2020 May 13.

14. Barrington-Trimis JL, Cho J, Ewusi-Boisvert E, Hasin D, Unger JB, Miech RA, Leventhal AM. Risk of persistence and progression of use of 5 cannabis products after experimentation among adolescents. JAMA network open. 2020 Jan 3;3(1):e1919792.

15. Martínez V, Iriondo De-Hond A, Borrelli F, Capasso R, del Castillo MD, Abalo R. Cannabidiol and Other Non-Psychoactive Cannabinoids for Prevention and Treatment of Gastrointestinal Disorders: Useful Nutraceuticals?. International Journal of Molecular Sciences. 2020 Jan;21(9):3067.

16. Almogi-Hazan O, Or R. Cannabis, the Endocannabinoid System and Immunity-the
Medico-legal Update, January-March 2021, Vol. 21, No. 1

91 Journey from the Bedside to the Bench and Back. International Journal of Molecular Sciences. 2020 Jan;21(12):4448.

17. Klein TW. Cannabinoid-based drugs as antiinflammatory therapeutics. Nature Reviews Immunology. 2005 May;5(5):400-11.

18. Pacher P, Bátkai S, Kunos G. The endocannabinoid system as an emerging target of pharmacotherapy. Pharmacological reviews. 2006 Sep 1;58(3):389462.

19. Nagarkatti P, Pandey R, Rieder SA, Hegde VL, Nagarkatti M. Cannabinoids as novel antiinflammatory drugs. Future medicinal chemistry. 2009 Oct;1(7):1333-49.

20. Klein TW, Newton CA, Nakachi N, Friedman H. $\Delta$ 9-tetrahydrocannabinol treatment suppresses immunity and early IFN- $\gamma$, IL-12, and IL-12 receptor $\beta 2$ responses to Legionella pneumophila infection. The Journal of Immunology. 2000 Jun 15;164(12):6461-6.

21. Lynn RS, Galinkin JL. Cannabis, e-cigarettes and anesthesia. Current Opinion in Anesthesiology. 2020 Jun 1;33(3):318-26.

22. Richardson S, Hirsch JS, Narasimhan M, Crawford JM, McGinn T, Davidson KW, Barnaby DP, Becker LB, Chelico JD, Cohen SL, Cookingham J. Presenting characteristics, comorbidities, and outcomes among 5700 patients hospitalized with COVID-19 in the New York City area. Jama. 2020 Apr 22. 